\title{
A Case Report on Acanthomatous Ameloblastoma of the Anterior Mandible with Brief Review on Advanced Imaging Diagnosis
}

\author{
Nivedita Chinam ${ }^{1}$ Aniket Vaidya ${ }^{1}$ Manisha Khorate ${ }^{1}$ Sonam Khurana ${ }^{2}$ \\ ${ }^{1}$ Department of Oral Medicine and Radiology, Goa Dental College and \\ Hospital, Bambolim, Goa, India \\ 2 Department of Comprehensive Dentistry, School of Dentistry, \\ University of Texas Health Science Center, San Antonio, Texas, \\ Address for correspondence Nivedita Chinam, MDS (Oral Medicine \\ and Radiology), Department of Oral Medicine and Radiology, Goa \\ Dental College and Hospital, Bambolim, Goa 403202, India \\ (e-mail: nivi0302@gmail.com).
} United States

Indian J Radiol Imaging 2021;31:1047-1052.

\begin{abstract}
Keywords

- ameloblastoma

- imaging

- neoplasm

- odontogenic tumor

Ameloblastoma is the most common benign odontogenic tumor of epithelial origin. It exhibits a locally aggressive behavior and high recurrence rate with multitude of factors involving in its molecular pathogenesis. This article reports a case of acanthomatous ameloblastoma involving the mandible in a 60-year-old male patient with peculiar imaging characteristics. The role of computed tomography and magnetic resonance imaging in diagnosis of conventional ameloblastoma has been elaborately emphasized in the discussion. Although the final diagnosis is based on histopathological features, physicians should be aware of the role of advanced imaging for diagnosis of ameloblastoma and for better surgical management.
\end{abstract}

\section{Introduction}

Ameloblastoma is benign odontogenic tumor with an aggressive growth tendency and a high potential for malignant transformation as well as metastasis. ${ }^{1}$ They represent $\sim 11$ to $18 \%$ of all odontogenic tumors and $1 \%$ of all oral tumors. ${ }^{2}$ First detailed description of ameloblastoma was given by Broca in the year 1868. He termed this lesion as odontomes embryoplastiques, which mean embryoplastic odontomas. ${ }^{3}$ It was designated as "adamantinoma" in 1885 by the French physician Louis-Charles Malassez. ${ }^{4}$ Churchill and Ivy described that the name adamantinoma indicates the neoplasm as calcified and hence coined the term ameloblastoma in $1930 .^{3,4}$ There is no predilection for sex. The peak incidence is in the third and fourth decades of life with the median age at presentation being 35.9 years and an age range of 4 to 92 years., Eighty percent of the cases develop in the mandible, most commonly in the vicinity of the molars and ramus. The ratio of maxillary and mandibular ameloblastomas is $1: 5.4{ }^{6}$ Clinically, it presents as a slow-growing relatively painless expansile tumor causing tooth mobility, tooth displacement, and a grotesque facial appearance if the patient delays getting treatment. ${ }^{1}$ Understanding the various radiographic characteristics is of crucial importance in the diagnosis of ameloblastoma. In the present case, we have considered magnetic resonance imaging (MRI) apart from the routine conventional radiographs and computed tomography (CT) scan, to well analyze the solid and cystic areas in the tumor mass. The objective of documenting this case is to emphasize on the imaging characteristics of conventional ameloblastoma.

\section{Case History}

A 60-year-old male patient presented to oral medicine and radiology department with a chief complaint of swelling in the anterior mandible region since 2 years. Patient had a published online

November 30, 2021
DOI https://doi.org/ $10.1055 / \mathrm{s}-0041-1739382$. ISSN 0971-3026.

\footnotetext{
(C) 2021. Indian Radiological Association. All rights reserved. This is an open access article published by Thieme under the terms of the Creative Commons Attribution-NonDerivative-NonCommercial-License, permitting copying and reproduction so long as the original work is given appropriate credit. Contents may not be used for commercial purposes, or adapted, remixed, transformed or built upon. (https://creativecommons.org/ licenses/by-nc-nd/4.0/)

Thieme Medical and Scientific Publishers Pvt. Ltd., A-12, 2nd Floor, Sector 2, Noida-201301 UP, India
} 


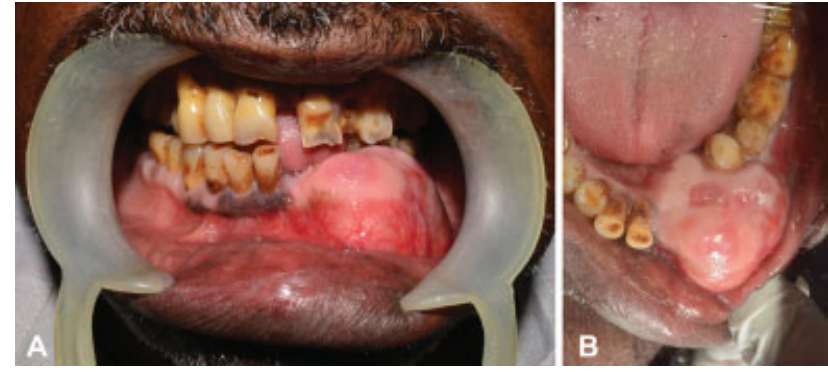

Fig. 1 (A) Intraoral photograph showing swelling on the buccal aspect in the anterior mandibular region. (B) Intraoral photograph showing occlusal view of the swelling.

history of extraction of lower left incisors and canine before the appearance of swelling and reported that the swelling began after extraction and did not subside since then. There were no other associated symptoms. Medical history revealed the patient was diabetic. On clinical examination, a solitary, hard painless swelling was evident over the anterior edentulous alveolar ridge, measuring $\sim 3 \times 3 \mathrm{~cm}$ in size extending from 3-5 to $4-1$ region. The swelling extended buccally to involve the buccal vestibule. The borders were well defined and overlying mucosa was smooth and pale pink with teeth indentations of the opposing tooth noted on the occlusal surface ( - Fig. 1). Tooth 3-4 was displaced lingually, 3-4 and 3-5 had grade II mobility, and 4-1 and 4-2 had grade I mobility. On pulp vitality test, 3-4 and 3-5 were nonvital. No sensory paralysis was observed. On aspiration with a fine needle, a blood tinged fluid was obtained (-Fig. 2). A provisional diagnosis of benign odontogenic tumor/cyst was considered.

Panoramic radiograph revealed the presence of a welldefined expansile radiolucency over the symphysis region extending from the 4-3 region till the mesial aspect of 3-6. Superiorly-inferiorly, it is seen extending from the alveolar crest till the superior margin of the inferior cortex of the mandible. Irregular areas of bony trabeculae were seen on the superior aspect suggestive of persistence of bone after destruction. Teeth $3-1,3-2$, and 3-3 were missing and tipping of 3-4 was evident ( - Fig. 3A). On intraoral periapical radiographs (3-6 to 4-2 region), a well-defined, thinly corticated, mixed density lesion was noted. This entity showed multiple small radiolucencies resembling a honey-

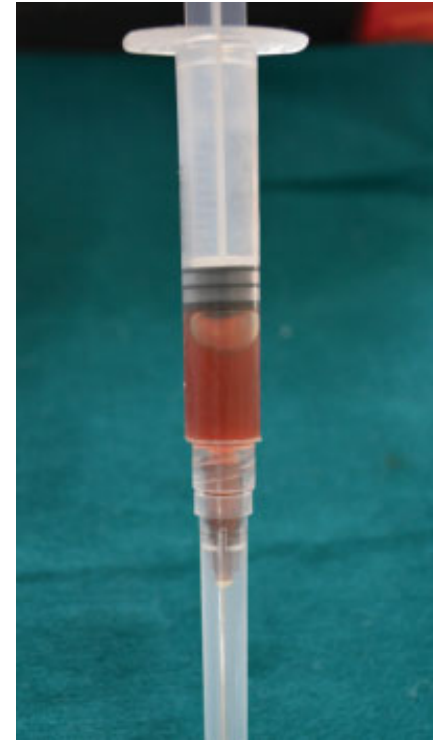

Fig. 2 Blood tinged aspirate obtained on aspiration.

comb pattern within the radiopaque altered trabeculae in the superior aspect of the lesion near the alveolar crest and a large undermining radiolucent lesion ( - Fig. 3B). Periodontal ligament space and lamina dura appeared to be effaced at the apex of the roots. On true occlusal radiograph $(-$ Fig. 3C), the lesion was seen involving entire marrow bone and causing nonuniform expansion, thinning and interruption of both buccal and lingual cortices with the buccal expansion greater than the lingual. A schematic representation of the lesion has also been depicted ( - Fig. 4).

Contrast-enhanced CT (CECT) scan demonstrated an expansile, well-defined, partially corticated, mixed density lesion in the anterior mandible region measuring $\sim 3.4 \times 3$ $\times 2 \mathrm{~cm}$ in size. Additionally, mental foramen was not traceable on the left side. On bone and soft tissue window without contrast, heterogeneous internal architecture was noted. High attenuation areas in the superior portion of the lesion were consistent with bony fragments ( $\mathbf{F i g . ~ 5 C , ~ D ) . ~}$ On soft tissue window with contrast, enhancing areas were noted consistent with the solid portion of the tumor and the nonenhancing content inferiorly was consistent with the cystic portion of the tumor (- Fig. 5A, B). Mean density values at the region of interest with bone fragments was
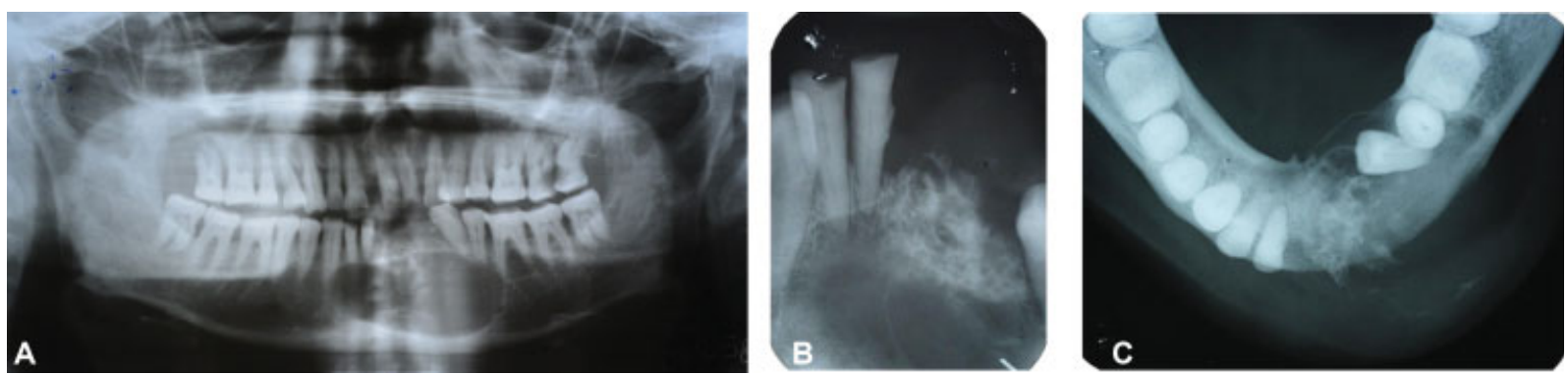

Fig. 3 (A) Orthopantomogram showing a radiolucency in the symphysis region extending from the 4-3 region till the mesial aspect of 3-6. (B) Intraoral periapical radiograph showing undermining cystic lesion with presence of altered trabecular pattern of the crestal alveolar bone in the region of 3-1, 3-2, and 3-3. (C) Mandibular true occlusal radiograph showing buccolingual extent of the lesion. 


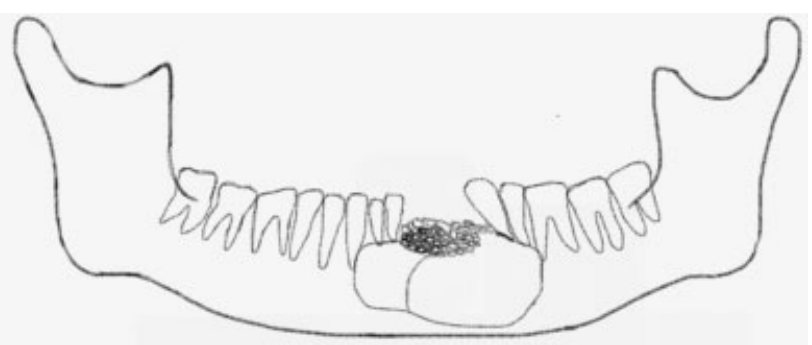

Fig. 4 Schematic diagram of pattern of ameloblastoma in the anterior mandible as seen in our case.

$642 \mathrm{HU}$ and the areas with low attenuation showed a mean density of $49 \mathrm{HU}$. MRI was acquired further to evaluate the internal contents of osteolytic lesion. Axial and coronal T1weighted (T1W) images were obtained at 3-mm-thick sections. T1W images (repetition time [TR] 676 and echo time [TE] 12) showed signal intensity isointense to muscle with minute areas of hypointensities. A portion of the lesion appeared hyperintense on T1W sequences suggestive of a subacute bleed ( - Fig. 6A, B). In T2-weighted (T2W) scan (TR 3890 and TE 99), the signal intensity was predominantly hyperintense indicative of cystic content. Fluid-fluid level was noted secondary to the cystic cavity being filled with blood products which settle to the gravity dependent area ( $\mathbf{F i g . ~ 6 C , ~ D ) . ~ S o l i d ~ p o r t i o n ~ o f ~ t h e ~ t u m o r ~ w a s ~ a l s o ~ a p p r e c i - ~}$ able in the medial aspect. Areas of low signal intensity were noted on the medial aspect which correspond to the areas of bone in CT scan and plain radiography (-Fig. $6 \mathbf{C C}$ ). The observations made in $\mathrm{T} 2 \mathrm{~W}$ sections confirmed that lesion is composed of both solid and predominantly cystic components.

Histopathology revealed a stroma showing presence of odontogenic epithelium as in the form of follicles of varying sizes. Follicles were lined by a single layer of tall columnar cells with a hyperchromatic nucleus polarized and palisaded away from the basement membrane (ameloblast-like cells), center of the follicles revealed stellate reticulum-like cells. A large number of follicles also showed squamous metaplasia and keratin pearl formation. Other follicles showed areas of cystic degeneration within the stellate reticulum-like cells. These features were consistent with acanthomatous ameloblastoma. Marginal mandibular resection was performed followed by stabilization with reconstruction plate (-Fig. 7). No recurrence was observed for 12 months after surgery.
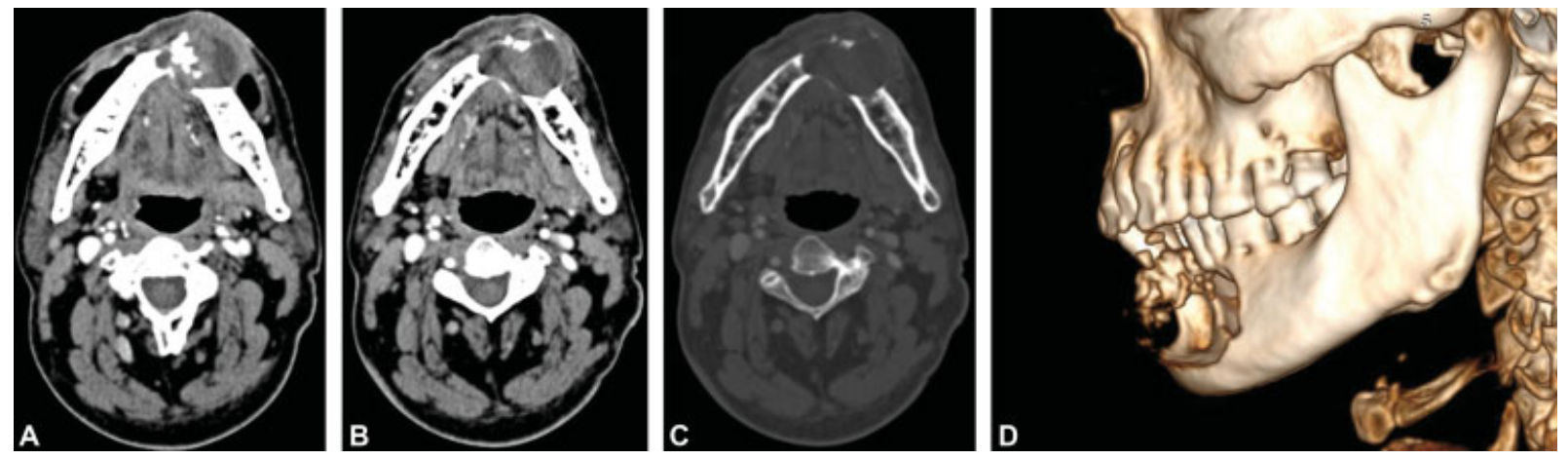

Fig. 5 (A) Contrast-enhanced computed tomography showing an expansile lytic lesion in the anterior mandible region extending from 43 to 35 region, with enhancing areas noted adjacent to the remnant bone. (B) The nonenhancing content inferiorly in the anterior mandible is suggestive of the cystic portion of the tumor. (C) Computed tomography scan of the osteolytic lesion in the anterior mandible as seen in the bone window. (D) Three-dimensional reconstructed image of lesion in the anterior mandible.
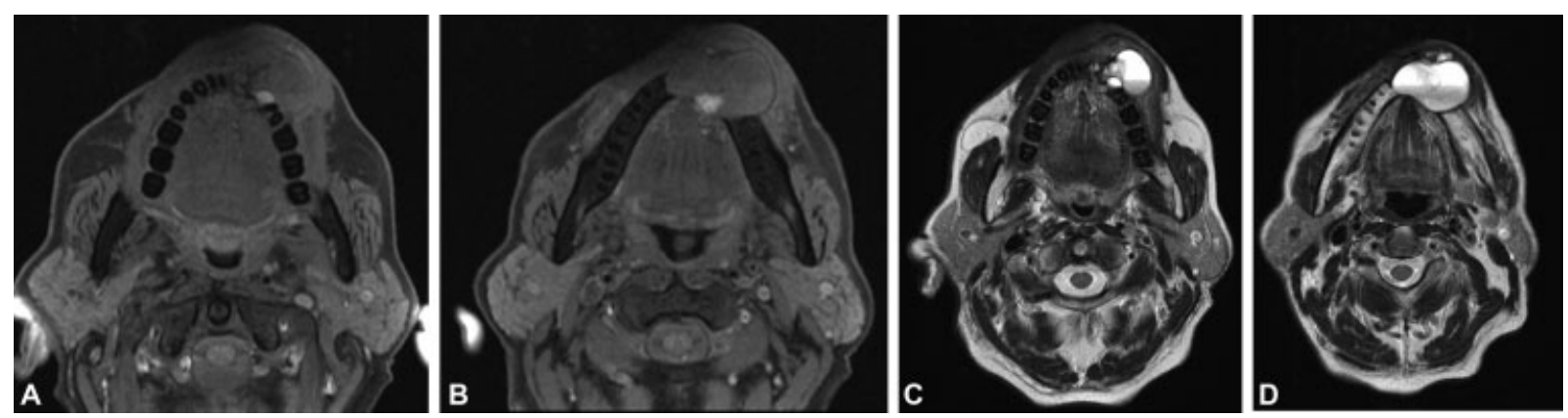

Fig. 6 (A) Magnetic resonance images: T1W images of the osteolytic lesion in the anterior mandible showing signal intensity isointense to muscle with minute areas of hypointensities. (B) Magnetic resonance images: T1W images of the osteolytic lesion in the anterior mandible showing signal intensity isointense to muscle with minute areas of hypointensities. A portion of the lesion appears hyperintense suggestive of a subacute bleed. (C, D) In T2W images of the osteolytic lesion in the anterior mandible, the signal intensity was predominantly hyperintense suggestive of a cystic lesion. Areas of intermediate and low signal intensity is noted on the medial aspect. Fluid-fluid level is noted within the cystic cavity. 


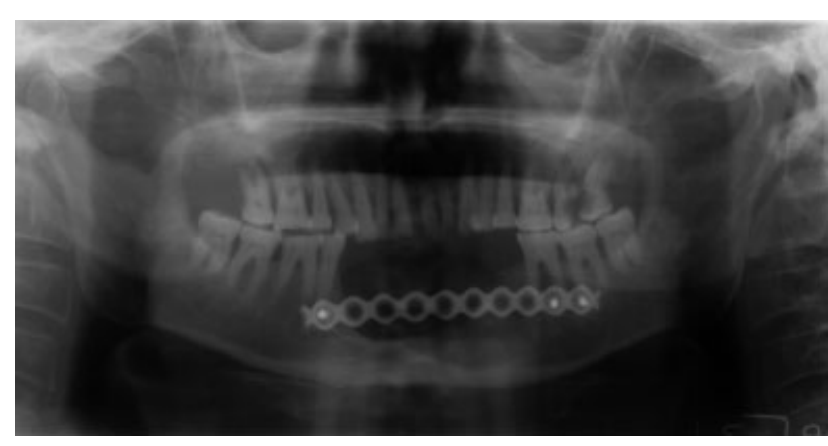

Fig. 7 Postoperative radiograph.

\section{Discussion}

Ameloblastoma is a true neoplasm of enamel organ type tissue which lacks the properties of odontogenic mesenchyme with no enamel formation due to impaired ameloblast function and absence of stratum intermedium cells. ${ }^{1}$ As per the fourth edition of the World Health Organization Classification of Odontogenic Tumors (2017), ameloblastoma has been classified as conventional, unicystic, extraosseous/peripheral types and metastasizing (malignant) ameloblastoma. The solid/multicystic type from the previous classification (2005) was eliminated because of its biologic insignificance and can be most likely confused with unicystic ameloblastoma. $^{7}$ The various histopathologic types are follicular, plexiform, desmoplastic, acanthomatous, granular cell, basaloid, clear cell, spindle, and hemangiomatous. Combinations of mixed histopathological patterns are also observed., ${ }^{1,2}$

The molecular pathogenesis involves activating mutations in cell signaling pathways such as mitogen-activated protein kinase (MAPK) which controls cellular processes such as cell proliferation and differentiation. BRAF V600E is the most common mutation implicated in the MAPK pathway and can be considered as a diagnostic marker to identify tumor progression. ${ }^{2}$ Non-MAPK signaling genes such as sonic hedgehog (SMO mutation) also play an important role in etiopathogenesis of ameloblastoma. ${ }^{1,7}$ Local invasiveness is the key biological feature of ameloblastoma which can be the plausible explanation for its high recurrence rate. ${ }^{5}$ Multitude of factors contribute to this property of ameloblastoma such as high levels of matrix metalloproteinases (MMP-9) which favor invasion by degrading collagen, structural components of the basement membrane and the extracellular matrix. ${ }^{2,5}$ Apart from this expression of diverse markers such as tissue inhibitor of metalloproteinase 2, E-cadherin and vimentin have also been implicated to its local aggressiveness. ${ }^{5}$ Several other studies have also shown dysregulation of key regulators in bone remodeling such as RANK, RANKL, and osteoprotegerin. ${ }^{1,2}$ All the above features make ameloblastoma an aggressive tumor with peculiar molecular characteristics. Fas, FasL (Fas ligand), caspase 3, p53, syndecan-1, Snail, and CD10 have been considered as the prognostic markers. ${ }^{2}$

In the imaging features, we will continue to use the terms solid/multicystic ameloblastoma as in the past literature, the same terminology is used. In a plain radiograph, the septae of ameloblastoma are usually coarse, curved, and originate from normal bone that has been trapped within the tumor. As the tumor grows, there may be coalescence and fusion of the compartments leading to transformation from a multilocular to a monolocular cystic space. H.M. Worth has described four patterns of radiographic appearances-unicystic type, spider-web pattern, soap-bubble pattern, honeycomb, or solid pattern. ${ }^{4}$ Among the histopathological variants mentioned, the desmoplastic variant presents with a distinctive mixed radiolucent-radiopaque radiographic pattern due to metaplastic bone formation. ${ }^{1}$ There are no significant differences in radiological appearance between the other pathological types of conventional ameloblastoma. In a clinicoradiographic study conducted on 73 cases of ameloblastoma in Indian population, multilocular radiolucency was predominant in follicular and acanthomatous ameloblastoma, while unilocular radiolucency and associated impacted teeth were more often seen in unicystic ameloblastoma. The acanthomatous variant showed a tendency to occur more commonly in the molar-ramus region and in males of older age group. ${ }^{8}$ However, there are no specific imaging features to differentiate the acanthomatous variant from others.

Advanced imaging modalities such as CT and MRI play a vital role in determining the margins and extent of the tumor. Noncontrast CT with soft tissue algorithm plays an important role in evaluation of the contents based on density values by comparing attenuation with surrounding muscle, while the use of a bone algorithm is recommended to clearly evaluate fine bony structures. ${ }^{9}$ Ameloblastoma shows cystic areas of low attenuation and isoattenuating solid regions on $\mathrm{CT}^{4}$ It has also been observed that the histopathological subtypes can produce different $\mathrm{CT}$ densities. Follicular ameloblastomas tend to show significantly lower mean internal density $(23.8 \mathrm{HU})$ than the plexiform variant $(37.7 \mathrm{HU}) .{ }^{10}$ However, no similar CT study has been reported in which all the pathological variants of conventional ameloblastoma are included. CECT scan shows an enhancement effect in the solid components. ${ }^{4}$ Angiogenesis in tumors plays a vital role in determining their appearance on CECT, and the timedependent changes in contrast enhancement resulting from tumor angiogenesis can be utilized to provide diagnostic information. Significant correlation between microvessel density (which depicts the vascularization of tumor tissue) and contrast enhancement characteristics in the $\mathrm{CT}$ scan has been reported in ameloblastoma. ${ }^{11}$ Hence, contrast enhancement is one of the important features for benign odontogenic tumors. As the pattern of bone destruction in the intraoral radiographs were suggestive of a tumor rather than a cyst, our aim initially to take CECT was to evaluate the enhancement pattern of the tumor. As the tumor revealed contrast enhancement in few areas, we further went on to take an MRI to better evaluate the internal architecture based on the T1W and $\mathrm{T} 2 \mathrm{~W}$ images.

MRI is the most useful modality for analyzing the internal structure as it exhibits a superior soft tissue contrast. ${ }^{4}$ It provides specific information about the solid and cystic 
components, irregular thickened walls, septa, papillary projections, and mural nodules. ${ }^{12}$ Solid type of ameloblastoma is a solid tumor which shows homogeneous low signal intensity on T1W image and homogeneous high signal intensity on T2W image. It indicates the presence of soft tissue, and shows strong enhancement. Multicystic type contains cysts of various sizes within solid tissue and is divided into solid and cystic portions on the basis of their signal intensities. The solid portions show low signal intensity on T1W image, high signal intensity on $\mathrm{T} 2 \mathrm{~W}$ image, and strong enhancement. The cystic portions show low signal intensity on T1W image, markedly high signal intensity on T2W image, with no enhancement. ${ }^{13}$ These multicystic features are due to the coalescence of microcysts formed due to degeneration of stellate reticulum cells within the tumor nests of columnar epithelium. ${ }^{1}$ Asaumi et al observed various patterns of enhancement with dynamic contrast-enhanced MRI which reflects the diversity of proliferation rate and hemodynamics per unit of ameloblastomas. However, no difference in contrast index curve patterns was observed among the various histopathological types. ${ }^{12}$ Unicystic ameloblastoma shows irregularly thick rim enhancement or intraluminal solid component, on CECT/contrast-enhanced MRI providing a clue to the correct diagnosis. ${ }^{9}$

CECT and MRI have also proven to be reliable in differential diagnosis of ameloblastoma. Apajalahti et al suggested that CECT and MRI enable differentiating nonunicystic ameloblastomas and odontogenic cysts because the latter do not show solid content and tend to show thin rim enhancement. ${ }^{9}$ The most important differential diagnosis emphasized in the literature is odontogenic keratocyst (OKC) as they share similar characteristics on conventional radiographs. The other differential diagnosis include odontogenic myxoma, central giant cell granuloma, aneurysmal bone cyst, and dentigerous cyst. Ameloblastoma shows a higher CT density than OKC as the former usually contains slightly proteinaceous fluids, occasionally associated with colloidal materials and cystic fluids of OLCs have a low soluble protein concentration. ${ }^{10}$ Higher heterogeneity values (variation of the average attenuation coefficient) was observed in OKC than ameloblastoma due to the presence of keratin. ${ }^{14}$ Dynamic contrast-enhanced MRI can be preferred to differentiate ameloblastoma from odontogenic myxoma. In ameloblastoma, the contrast agent does not enter the cystic portion but rapidly enters the tumor substance, while in myxoma, it enters the central portion, which appears to be a cyst-like area and shows a strong peripheral rim enhancement. ${ }^{12}$ Apart from this, Konouchi et al put forth a four-step diagnostic protocol for diagnosing ameloblastoma, OKC, dentigerous cyst, and simple bone cyst based on contrast-enhanced MRI and diffusion-weighted imaging. ${ }^{13}$

Management of ameloblastoma can be done through three modalities (1) conservative which includes enucleation and curettage and the use of adjuvant therapies such as Carnoy's solution and cryotherapy, (2) marsupialization, and (3) radical surgery consists of marginal or block resection (1-1.5 cm margins result in the highest chance of local control) and immediate bone reconstruction. Facial recon- struction procedures with iliac crest grafts or microvascular fibular flaps may be required. ${ }^{6,15}$ Radiotherapy should be considered in patients with positive margins in whom reresection is not recommended and for those with incompletely resectable tumors. The dose fractionation ranges from $66 \mathrm{~Gy}$ in 33 once-daily fractions for microscopic residual disease to $70 \mathrm{~Gy}$ in 35 once-daily fractions or $74.4 \mathrm{~Gy}$ in 62 twice-daily fractions for gross disease. ${ }^{6}$ Molecular-targeted therapies have been developed which include therapeutic drugs such as denosumab, vemurafenib, perifosine, cetuximab, and trastuzumab. Recurrence rates of 50 to $90 \%$ have been reported after curettage and up to $15 \%$ after marginal or block resection for conventional ameloblastomas. $^{2}$

\section{Conclusion}

Ameloblastoma is the most common odontogenic tumor in the oral cavity and can show polymorphic presentations. Acanthomatous ameloblastoma is a rare variant. Although there are multiple case reports of acanthomatous ameloblastoma reported in the literature, our case is unique as it was noted in the anterior mandible, and the CECT and MRI features have been described in detail, which show a predominantly cystic lesion. In our opinion, it is prudent that we know and understand the various intricate imaging features so as to distinguish ameloblastoma from other tumors.

\section{Declaration of Patient Consent}

The authors certify that they have obtained all appropriate patient consent forms. In the form, the patient(s) has/have given his/her/their consent for his/her/their images and other clinical information to be reported in the journal. The patients understand that their names and initials will not be published and due efforts will be made to conceal their identity, but anonymity cannot be guaranteed.

\section{Financial Support and Sponsorship \\ Nil.}

\section{Conflict of Interest}

None declared.

\section{References}

1 Effiom OA, Ogundana OM, Akinshipo AO, Akintoye SO. Ameloblastoma: current etiopathological concepts and management. Oral Dis 2018;24(03):307-316

2 Jhamb T, Kramer JM. Molecular concepts in the pathogenesis of ameloblastoma: implications for therapeutics. Exp Mol Pathol 2014;97(03):345-353

3 Yoithapprabhunath TR, Nirmal RM, Ganapathy N, et al. Metaterminology of ameloblastoma. J Pharm Bioallied Sci 2019;11 (Suppl 2):S140-S145

4 More C, Tailor M, Patel HJ, Asrani M, Thakkar K, Adalja C. Radiographic analysis of ameloblastoma: a retrospective study. Indian J Dent Res 2012;23(05):698

5 Florescu A, Mărgăritescu C, Simionescu CE, Stepan A. Immunohistochemical expression of MMP-9, TIMP-2, E-cadherin and vimentin in ameloblastomas and their implication in the local 
aggressive behavior of these tumors. Rom J Morphol Embryol 2012;53(04):975-984

6 Mendenhall WM, Werning JW, Fernandes R, Malyapa RS, Mendenhall NP. Ameloblastoma. Am J Clin Oncol 2007;30(06): 645-648

7 Wright JM, Vered M. Update from the 4th edition of the World Health Organization classification of head and neck tumours: odontogenic and maxillofacial bone tumors. Head Neck Pathol 2017;11(01):68-77

8 Krishnapillai R, Angadi PV. A clinical, radiographic, and histologic review of 73 cases of ameloblastoma in an Indian population. Quintessence Int 2010;41(05):e90-e100

9 Apajalahti S, Kelppe J, Kontio R, Hagström J. Imaging characteristics of ameloblastomas and diagnostic value of computed tomography and magnetic resonance imaging in a series of 26 patients. Oral Surg Oral Med Oral Pathol Oral Radiol 2015;120 (02):e118-e130

10 Ariji $\mathrm{Y}$, Morita M, Katsumata A, et al. Imaging features contributing to the diagnosis of ameloblastomas and keratocystic odonto- genic tumours: logistic regression analysis. Dentomaxillofac Radiol 2011;40(03):133-140

11 Hayashi K, Tozaki M, Sugisaki M, Yoshida N, Fukuda K, Tanabe H. Dynamic multislice helical CT of ameloblastoma and odontogenic keratocyst: correlation between contrast enhancement and angiogenesis. J Comput Assist Tomogr 2002;26(06):922-926

12 Asaumi J, Hisatomi M, Yanagi Y, et al. Assessment of ameloblastomas using MRI and dynamic contrast-enhanced MRI. Eur J Radiol 2005;56(01):25-30

13 Konouchi H, Yanagi Y, Hisatomi M, et al. MR imaging diagnostic protocol for unilocular lesions of the jaw. Jpn Dent Sci Rev 2012; 48:81-91

14 Crusoé-Rebello I, Oliveira C, Campos PS, Azevedo RA, dos Santos JN. Assessment of computerized tomography density patterns of ameloblastomas and keratocystic odontogenic tumors. Oral Surg Oral Med Oral Pathol Oral Radiol Endod 2009;108(04):604-608

15 Hertog D, van der Waal I. Ameloblastoma of the jaws: a critical reappraisal based on a 40-years single institution experience. Oral Oncol 2010;46(01):61-64 